\title{
The Phenomenology of Spatial Integration: Data and Models
}

\author{
Geoffrey R. Loftus and Aura M. Hanna \\ University of Washington
}

\begin{abstract}
A briefly presented visual stimulus followed by darkness seems to persist beyond its physical offset. We are concerned here with the relation between two characteristics of this visible persistence: first, its phenomenological resemblance to the stimulus that spawned it and second, its usefulness as a basis for integrating visual stimuli that are separated in time. We describe two experiments using a task in which two halves of a visual stimulus were presented successively and observers reported how complete the stimulus appeared to be. Stimuli appeared less complete with increases in both the duration of the interval intervening between presentation of the two halves and the duration of the initially presented stimulus half. This data pattern is similar to that obtained in tasks in which spatial integration of two temporally disparate stimuli is necessary for correct responding. On the basis of this similarity, we argue that phenomenological appearance and ability to integrate stimuli over time are two facets of the same perceptual events. We describe a formal model to account for these and other data. (1) 1989 Academic Press, Inc.
\end{abstract}

A bricfly presented visual stimulus followed by darkness seems to persist beyond its physical offset. The mental representation that corresponds to this poststimulus perceptual activity has two salient characteristics. First, the representation looks like a literal (albcit a fading) cxtension of the stimulus that it follows. Second, the representation can be used as a basis for performing tasks that require knowing the spatial relations of features within the stimulus. Such tasks include temporal integration (e.g., Di Lollo, 1980; Eriksen \& Collins, 1967) and partial report (e.g., Di Lollo \& Dixon, in press; Sperling, 1960.)

This article is concerned with both empirical investigation of persistence and with theoretical accounts of it. Accordingly, the article is divided into two major sections. In the first section, we describe a new method for investigating visible persistence. In particular, we are con-

The research described in this article was supported by an NIMH grant to Geoffrey Loftus. We thank Vince Di Lollo, Pete Dixon, Tony Greenwald, Dave Irwin, Beth Loftus, John Palmer, and Karen Preston for helpful comments on earlier versions of this manuscript, and on the research issues under consideration. Requests for reprints should be sent to Geoffrey R. Loftus, Department of Psychology, University of Washington, Seattle, WA 98195. 
cerned with effects on a stimulus's phenomenological presence of three variables: stimulus duration, time since stimulus offset, and stimulus complexity. In the second section, we describe a picture-perception model developed by Loftus and Hogden (1988) and show how it accounts for our data as well as for partial-report data reported by Di Lollo and Dixon (in press). We compare this model to a model presented by Di Lollo and Dixon and show that there is a good deal of formal similarity between them.

To provide a foundation for discussing these issues we will start by briefly describing some characteristics of visible persistence, and some models that have been developed to account for the characteristics.

\section{PERSISTENCE AS A BASIS FOR PHENOMENOLOGICAL EXPERIENCE}

The visible persistence that follows a briefly presented stimulus seems like a genuine extension of the physical stimulus. Indeed, naive observers think that visible persistence is an extension of the physical stimulus. When shown a 10-ms stimulus followed by darkness, for example, observers often express the belief that the projector bulb responsible for displaying the stimulus is extinguishing slowly, and they are subsequently astonished to learn that what they had seen existed only in their minds.

The time course of visible persistence can be investigated in a variety of ways. A common technique is the synchrony-judgement task, wherein a briefly presented stimulus is followed after its offset by a variable interval, and then by some salient synchrony signal, such as an auditory click. The variable interval is under the observer's control, and the observer's task is to adjust the interval's length such that the synchrony signal seems to just coincide with the stimulus's complete disappearance. The interval duration that satisfies this requirement constitutes an estimate of persistence duration. Investigators using this technique have found persistence duration to be an inverse function of stimulus duration (e.g., Efron, 1970a, b; Loftus \& Hogden, 1988) and usually (but not always) an inverse function of stimulus luminance (Bowen, Pola, \& Matin, 1974; Sakitt, 1976; see Coltheart, 1980, for a review of both duration and luminance effects).

\section{PERSISTENCE AS A BASIS FOR PRESERVING SPATIAL INFORMATION}

The mental representation that corresponds to visible persistence can be used to perform certain tasks that require knowledge of spatial relations within the stimulus. A common such task is the temporal-integration task reported first by Eriksen and Collins (1967). In a temporal-integration task, two halves of a stimulus are presented in succession. Each stimulus 
half is itself meaningless. However, the halves are spatially related such that, when spatially superimposed, they form a meaningful pattern, some aspect of which must be reported by the observer. For example, Eriksen and Collins used stimuli whose halves looked like random-dot patterns, but which, when superimposed, formed letter strings.

The experiments that we will report in this article are based on a variant of a temporal-integration task called the missing-dot task, which was developed by Di Lollo and his colleagues (e.g., Di Lollo, 1980; Di Lollo \& Woods, 1981; Hogben \& Di Lollo, 1974). The basic stimulus in the missing-dot task is an array of 24 dots that occupy 24 of the 25 cells in an imaginary $5 \times 5$ grid. Thus, one of the cells is missing a dot, and the observer's task is to report that cell's location. Performance is virtually perfect when all 24 dots are presented simultaneously; the question is, how good is performance when the 24 dots are not presented simultaneously?

To answer this question, the 24-dot array is randomly divided into two 12-dot stimulus halves. On each of a series of trials, the two halves, half-1 and half-2, are presented successively, and the observer's task is to report the location of the missing-dot cell. Performance under such circumstances declines as half-1/half-2 interstimulus interval (ISI) increases (Di Lollo \& Woods, 1981; Hogben \& Di Lollo, 1974). Holding all other relevant factors at their optimal levels, performance asymptotes when ISI reaches about $150 \mathrm{~ms}$.

This effect makes sense; as the time since the offset of the physical stimulus increases, stimulus visibility decreases, and the intrinsically visual missing-dot task becomes more difficult. Somewhat less intuitive, however, is the finding that the duration of the first half affects performance just as strongly as does ISI. Di Lollo (1980) found that, with a constant ISI of $10 \mathrm{~ms}$, performance dropped from virtually perfect to asymptote as half-1 duration (H1D) increased from 10 to about $150 \mathrm{~ms}$.

\section{MODELS OF VISIBLE PERSISTENCE}

A variety of models have emerged to account for the various facets of visible persistence (e.g., Erwin, 1976; Rumelhart, 1969; Sperling, 1967). It is beyond the scope of this article to provide a complete review of these models. Several models, however, are quite relevant to the one that we will later describe and we briefly sketch them here.

\section{The Recruiting/Interpreting Model}

Di Lollo (1980) outlined a model to account for performance in the missing-dot task. An observer in this task has a clear intuition about the deleterious effects of both ISI and H1D in temporal-integration tasks: they have to do with perceptual grouping of the halves. At long ISIs, the 
two halves appear to be two separate stimuli, and thus the visual integration necessary to identify the missing-dot location is not possible. Similarly, with long H1Ds, half-1 processing seems complete at the time of half-2 onset; thus the half- 2 display seems like an entirely new visual event. It is only with both a short HID and a short ISI that the two halves appear to constitute a unified stimulus, and thereby permit an accurate response.

Di Lollo's model assumes that encoding of a visual stimulus consists of a recruiting phase, followed by an interpreting phase. The recruiting phase is assumed to begin at stimulus onset, and involves encoding of the stimulus in terms of "features, such as dots, bars, edges, and discontinuities" (p. 93). The subsequent interpreting phase involves identification and categorization, i.e., the creation of a more permanent memory representation (see also Intraub, 1985; Loftus, Hanna, \& Lester, 1988; Potter, 1976). Of primary importance in explaining missing-dot performance is the nature of the recruiting phase, which involves maintenance of original stimulus geometry, and constitutes visible persistence. Di Lollo explicitly links missing-dot performance to recruitment activity; he asserts that "visual persistence is regarded ... as the product of the activity of sensory coding mechanisms engaged in the formation of "feature-encoded' stimuli." Thus, as soon as the recruitment activity is completed, persistence ends. Because recruitment activity begins at stimulus onset, the end of recruitment depends on time since stimulus onset, i.e., on stimulus onset asynchrony (SOA). The longer the SOA, the more likely it is that recruitment activity will have ended and, in accord with the data, the poorer will be missing-dot performance. SOA is, of course, the sum of H1D and ISI; thus it increases with increases in either of these variables.

\section{The Persistence/Analog Representation Model}

The simplest version of Di Lollo's model was disconfirmed by partialreport data which demonstrated visible persistence that seemed to depend on stimulus offset rather than on stimulus onset (Irwin \& Brown, in press; Irwin \& Yeomans, 1986; Loftus, 1985; Loftus \& Hogden, 1988). To explain this finding, Irwin and his colleagues described a new model in which spatial performance is determined by two things: visible persistence and a nonvisible, but spatial-information preserving analog representation. In this model, visible persistence is time-locked to stimulus onset, whereas the visual analog representation is time-locked to stimulus offset. ${ }^{1}$

${ }^{1}$ This model also accounts for the finding that asymptotic performance in the missing-dot paradigm is substantially above chance. 
Di Lollo and Dixon (in press) formalized Irwin's model as a set of equations describing the time courses of the two processes. We will describe this formalization, along with the application of the model to data in a later section of this article.

\section{The Information-Extraction Rate Model}

Loftus and Hogden (1988) describe a model that borrows from Di Lollo (1980) and is similar in important respects to the Irwin/Di Lollo and Dixon model. In the second section of this article, we present this model and its application to data in detail; hence we defer a formal, mathematical description of it. In this section, we present a bricf description of the model's central assumptions, and we sketch how the model makes a prediction about the effect of visual complexity on temporal-integration performance.

The model: A brief description. The model assumes that information from a visual stimulus is extracted at some rate. The informationextraction process, which occurs both during stimulus presence and for a short time following stimulus offset, corresponds roughly to the recruiting phase in Di Lollo's (1980) model. The magnitude of the informationextraction rate depends on two factors: rate increases as a function of the amount of available, extractable information and decreases as a function of the amount of information already extracted from the stimulus. The magnitude of visible persistence-which determines missing-dot performance-is determined by the information-extraction rate. The model accounts for both the HID and the ISI effects on missing-dot performance as follows. The information-extraction rate decreases with H1D because the amount of extracted half- 1 information increases with H1D. The information-extraction rate decreases with ISI because the amount of available information decreases with ISI and, in addition, because the amount of extracted half- 1 information increases with ISI. Therefore, according to the model, missing-dot performance must decline with both HID and with ISI.

Stimulus complexity. In the preceding section, we asserted that the information-extraction rate is influenced by amount of information already extracted from the stimulus. This characterization was for ease of exposition. More precisely, the information-extraction rate is assumed by the model to depend on amount of as-yet-unextracted stimulus information. This facet of the model allows a prediction about stimulus complexity which is derived more formally later in this article. Informally it is as follows. At any given time since stimulus onset, more as-yet-unextracted information remains in complex, relative to simple stimuli. Therefore, the decline in information-extraction rate with time since stimulus onset is less for complex, relative to simple stimuli. This in turn means that the 
decline in temporal-integration ability with time since stimulus onset is less with complex, relative to simple stimuli. ${ }^{2}$ This prediction was tested in the present experiments.

\section{EXPERIMENTS}

We report two experiments using a task that is similar to the missingdot task. We call this task the subjective-completeness task. Whereas the missing-dot task measures objective spatial-integration ability, the subjective-completeness task measures a fundamentally phenomenological event: how complete a visual stimulus appears to be. In this task, a visual stimulus is randomly divided into two halves, which are presented successively, and the observer's task is to rate how much the successively presented halves resemble the complete picture.

The major purpose of our experiments was to test the proposition that the phenomenological experience of stimulus completeness and spatialintegration ability are two manifestations of the same fundamental perceptual events. Accordingly, we examined rated completeness as functions of both H1D and ISI. If this proposition is true, then we should find the same data pattern with completeness rating as has been found with spatial integration: rated completeness should decrease with both H1D and ISI.

An additional purpose of the experiments was to test the prediction that the effect of H1D and ISI on persistence is greater for simple, relative to complex stimuli. To accomplish this, we included two types of stimuli in the experiment. Complex stimuli consisted of naturalistic, color scenes. Simple stimuli consisted of an array of dots.

\section{Experiment 1}

Experiment 1 consisted of a $6(\mathrm{H} 1 \mathrm{D}) \times 6$ (ISI) $\times 2$ (stimulus type: scenes or dots) factorial design. H1D ranged from 20 to $200 \mathrm{~ms}$, and ISI ranged from 0 to $160 \mathrm{~ms}$.

\section{Method}

Experiments 1 and 2 were quite similar. We described the Expriment 1 methodology here in detail, and subsequently describe only the Experiment 2 methodology that is different.

Observers. Ten members of the University of Washington Psychology Department participated. All except two (the authors) were paid $\$ 5$ per session for one practice session and two experimental sessions. Each session lasted approximately $1 \mathrm{~h}$.

Stimuli. Five colored scenes, four landscapes and one portrait of an old man, were selected from a travel brochure. A sixth picture consisted of a $4 \times 4$ array of dots. Each picture was divided into $4 \times 4$-in. grid, and can thus be conceptualized as consisting of

\footnotetext{
${ }^{2}$ It should be noted that this effect is predicted by Di Lollo's (1980) model, as well.
} 
16-in-square cells. The entire picture subtended $16^{\circ} \times 16^{\circ}$ of visual angle; thus, each cell subtended $4^{\circ} \times 4^{\circ}$. Each dot subtended $2^{\circ} \times 2^{\circ}$ visual angle, and was centered in its cell.

Six halvings were created, where a halving is a random division of a 16-cell picture into two 8-cell halves. These six halvings were applied to all six pictures; that is, each of the six pictures was halved in six different ways. We refer to each picture/halving combination as a stimulus; thus, there were 6 scenes $\times 6$ halvings or 36 stimuli in all. Note that each stimulus consists of two 8-cell, spatially complementary halves, which we refer to as half-A and half-B. Each of the 72 halves was prepared as a $35-\mathrm{mm}$ slide with the eight nonvisible cells blacked out. Each stimulus, therefore, was constructed such that displaying its two halves simultaneously (each half from a separate projector) resulted in the complete picture's appearance.

Apparatus. Stimulus halves were displayed via Kodak carousel projectors, and subtended horizontal and vertical visual angles of $15^{\circ}$. Timing was controlled by Gerbrands tachistoscopic shutters with rise and fall times of approximately $1 \mathrm{~ms}$. A third projector was used to present a dim fixation point that initiated each trial. ${ }^{3}$ All display equipment was enclosed in a soundproof box. Responses were collected on a 16-key response box. All display and response-collection equipment was controlled by an Apple II computer system described by Loftus, Gillispie, Tigre, and Nelson (1984).

Design and procedure. An experimental session consisted of a series of trials. Each trial consisted of a display followed by an immediate response.

The display consisted of two halves of a stimulus pair, usually presented successively. The first half's duration was $20,40,80,120,160$, or $200 \mathrm{~ms}$. The second half's duration was always $20 \mathrm{~ms}$. The ISI between the two halves was $0,40,80,120$, or $160 \mathrm{~ms}$. In a sixth ISI level, the two halves were presented simultaneously rather than successively. In this ISI level, both halves-i.e., the complete stimulus-was presented for one of the six H1Ds. Thus, all six ISI levels were factorially combined with the six H1D levels for a total of 36 within-subjects display conditions.

Following each display, the observer provided a rating ranging from 1 to 4 of how complete, or integrated, the picture seemed. Observers were instructed that the simultaneous control conditions represented an anchor point; i.e., that pictures in these conditions looked as complete as they ever would, and should therefore be assigned the maximum completeness rating of " 4 ." They were further instructed to assign a completeness rating of " 1 " to a picture whose two halves seemed entirely separate in time. Finally, they were instructed to appropriately assign the " 2 " and " 3 " intermediate ratings to intermediate perceptions.

Each of the 36 total stimuli was presented twice in each of the 36 display conditions: once with half- $A$ shown first in the display sequence, and again with half-B shown first. Thus, cach observer saw a total of 36 (stimuli) $\times 36$ (display conditions) $\times 2$ (half-A tcmporally first/second) $=2592$ trials.

These trials were grouped into 36-trial blocks. Each block incorporated a single stimulus (i.e., a single picture, halved in a particular way) shown once in each of the 36 display conditions. At the beginning of each block, the complete (i.e., both halves) picture for that block was displayed for several seconds. The observer inspected it while, if necessary, the experimenter fine-adjusted the projector alignments. The stimulus then appeared in 36 successive trials, one in each of the 36 display conditions. Six blocks, or a superblock, constituted all six original pictures (the five scenes plus the dots). Six superblocks, or a session, constituted all 36 stimuli (the six pictures rotated through the six halvings) in all experimental conditions. The second session was a replication of the first session, but with the half-A/half-B presentation orders reversed.

\footnotetext{
${ }^{3}$ Stimulus luminances (in millilamberts) were as follows: adapting field, 0.07 ; projector on, no slide, 38.43 ; and fixation spot, 0.38 . The dot stimuli were black ( 2.57 millilamberts) on a white background ( 25.19 millilamberts).
} 
The condition order was randomized for each block, and the order of the six pictures was randomized for each superblock. The half- $A /$ half-B presentation order was randomly determined on cach trial during the first session; these orderings then determined the corresponding orderings (which were reversed) during the second session.

Each observer participated in a complete session (1298 trials) as practice, prior to beginning the two experimental sessions.

\section{Results and Discussion}

Figure 1 shows mean completeness rating as a function of H1D; a separate curve is drawn for each of the six ISI levels. The left panel shows performance averaged over the five scenes, and the right panel shows performance for the dots. The standard errors were 0.053 and 0.070 for scenes and dots, respectively. We do not show these standard errors on the figures, because they are approximately the size of the curve symbols.

Normal (temporally disjoint) conditions. It is evident that rated completeness for both scenes and dots declines as a function of both ISI and H1D.

Simultaneous-presentation conditions. As expected from the instructions, the ratings from the simultaneous control conditions (top curves) do not depend on H1D, and are virtually at ceiling.

Scenes vs dots. Ratings range from ceiling to floor over the 36 conditions for both types of pictures. But does scene and dot performance differ with respect to dependence on ISI and H1D? Figure 2 compares the scene and dot performance decreases over H1D (left) and ISI (right). In both panels, three pairs of curves are shown: scene and dot performance for the smallest value of the factor not shown on the abscissa (0-ms ISI or
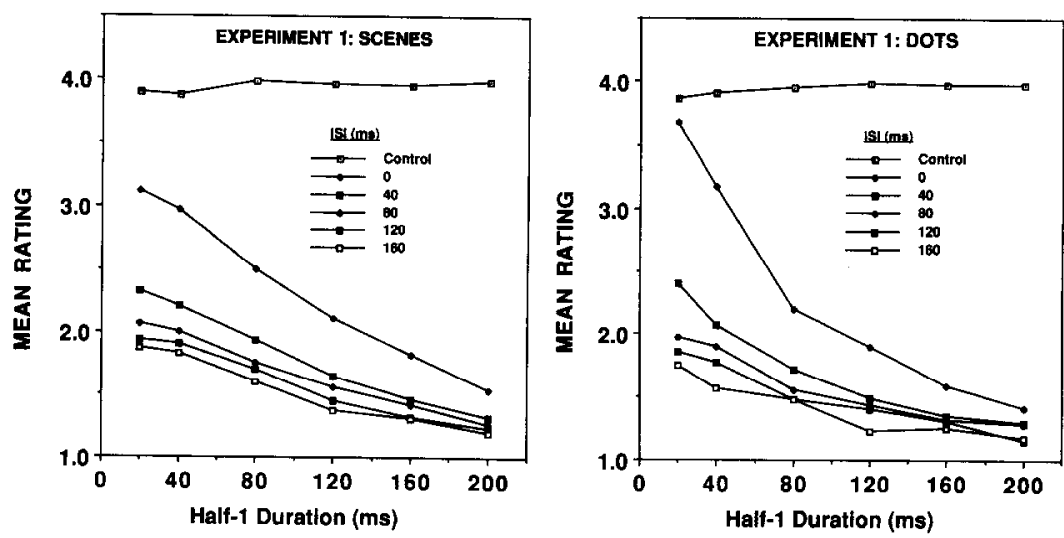

Fig. 1. Experiment 1: Mean rating as functions of half-1 duration. The curve parameter is interstimulus interval. The left panel shows data for scenes; the right panel shows data for dots. Each scene data point is based on $\mathbf{2 1 6 0}$ observations, and each dot data point is based on 432 observations. 

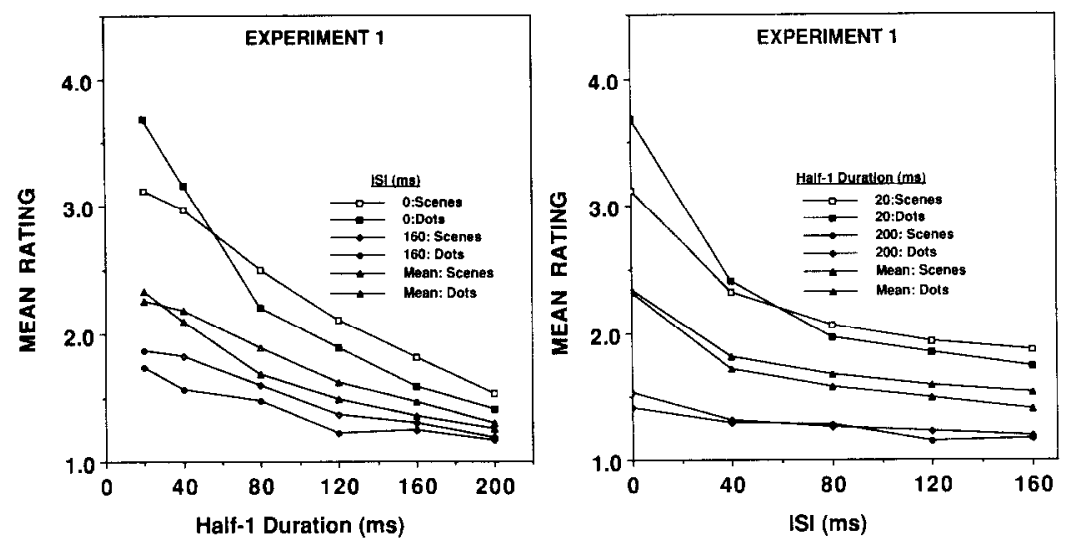

FIG. 2. Experiment 1: The scene/dot $\times$ half-1 duration interaction for different levels of interstimulus interval (left) and the scene/dot $\times$ interstimulus interval interaction for different levels of half- 1 duration (right).

20-ms H1D), for the largest value of the nonabscissa factor (160-ms ISI or 200-ms H1D), and collapsed over the nonabscissa factor (ISI and H1D for the left and right pancls respectively). It is evident that when the value of either factor is small, dot performance decreases faster than scene performance as a function of the other factor. However, the scene/dot interaction with either factor disappears when the value of the other factor is large.

To assess the interactions of stimulus type with H1D and ISI in more detail, we performed six 2 (scene/dot) $\times 5$ (ISI) ANOVAs: one ANOVA for each value of H1D. Similarly, we performed five 2 (scene/dot) $\times 6$ (H1D) ANOVAs: one ANOVA for each value of ISI (we excluded the simultaneous-presentation ISI level). The results of these ANOVAs, in the form of interaction $F$ values are shown in Table 1 . The scene/dot $\times$ ISI

TABLE 1

Experiment 1: $F$ Ratios for Dot/Scene (D/S) Interactions with Half-1 Durations and ISIs

\begin{tabular}{ccccc}
\hline \multicolumn{2}{c}{$\begin{array}{c}\text { D/S } \times \text { ISI interaction as a } \\
\text { function of half-1 duration }\end{array}$} & & \multicolumn{2}{c}{$\begin{array}{c}\mathrm{D} / \mathrm{S} \times \text { half-1 duration interaction } \\
\text { as a function of ISI }\end{array}$} \\
\cline { 1 - 2 } Half-1 duration (ms) & $F(4,36)$ & & ISI (ms) & $F(5,45)$ \\
\hdashline 20 & 12.10 & & 0 & 13.75 \\
40 & 4.94 & & 40 & 3.66 \\
80 & 1.55 & 80 & 1.52 \\
120 & 0.88 & 120 & 1.09 \\
160 & 1.09 & 160 & 2.03 \\
200 & 0.87 & &
\end{tabular}

Note. D/S $\times$ ISI interaction is based on 5 ISIs: $0-160 \mathrm{~ms}$. Criterion $F(\alpha=0.05)=2.82$ and 2.43 for $(4,36)$ and $(5,45)$ degrees of freedom, respectively. 
interaction is significant only for the 20 - and 40-ms H1D levels. Similarly, the scene/dot $\times$ H1D interaction is significant only for the 0 - and 40-ms ISI levels.

\section{Experiment 2}

Experiment 2 was designed principally to replicate the major findings of Experiment 1 under slightly different experimental conditions. Because much of the rating variation occurred at fairly short H1Ds and ISIs, we used generally shorter values of these variables in Experiment 2. A second purpose of Experiment 2 was prompted by indirect cvidence reported by Di Lollo (1980, Experiment 5) that the deleterious effect of increasing H1D obtains even when the two stimuli temporally overlap. To investigate this finding, we included two temporal-overlap ISI levels: one in which the onsets of the two halves always coincided, and another in which the offsets of the two halves coincided. Half-2 duration was always $20 \mathrm{~ms}$.

\section{Method}

The Experiment 2 method was similar, in most respects, to the Experiment 1 method.

Observers, stimuli, and apparatus. The 10 observers from Experiment 1 participated in Experiment 2. Again, all observers except the authors were paid \$5 per session for participating in three, 1-h sessions. The stimuli and apparatus were the same as in Experiment 1.

Design and procedure. The Experiment 2 procedures were similar to the Experiment 1 procedures. In Experiment 2, there were 35 display conditions, defined by five H1D levels factorially combined with seven ISI levels. The H1Ds were $20,40,60,80$, or $100 \mathrm{~ms}$. The half-2 duration was always $20 \mathrm{~ms}$. In five of the seven ISI levels the two stimulus halves were, as usual, temporally disjoint, with ISIs of $0,20,40,60$, and $80 \mathrm{~ms}$. In the remaining two ISI levels, the first and second halves temporally overlapped. In the synchronized-onset conditions, the onsets of the first and second halves coincided, and in the synchronizedoffset conditions, the offsets of the first and second halves coincided. Note that the synchronized-offset condition corresponds to ISI $=-20 \mathrm{~ms}$. Thus, the synchronized-offset condition can be considered as being on the same ISI scale as the temporally disjoint conditions; the only difference is that ISI is negative rather than nonnegative. Note also that 2 of the 35 conditions-20-ms half-1/synchronized onset, and $20-\mathrm{ms}$ half-1/synchronized offset-were identical (although they were distinct from a design perspective). In these two conditions, the complete picture was shown for $20 \mathrm{~ms}$.

As in Experiment 1, each of the 36 stimuli was shown twice in each of the 35 conditions: once with half-A shown first in the display sequence, and again with half-B shown first. Thus, each observer saw a total of 36 (stimuli) $\times 35$ (display conditions) $\times 2$ (half-A temporally first/second) $=2520$ trials. As in Experiment 1, these trials were grouped into 35-trial blocks which, in turn, were grouped into six-block superblocks. Randomization and counterbalancing were the same as in Experiment 1. As in Experiment 1, each observer participated in a practice session (1260 trials) prior to beginning the two experimental sessions.

\section{Results and Discussion}

Figure 3, which is organized like Fig. 1, shows mean completeness 

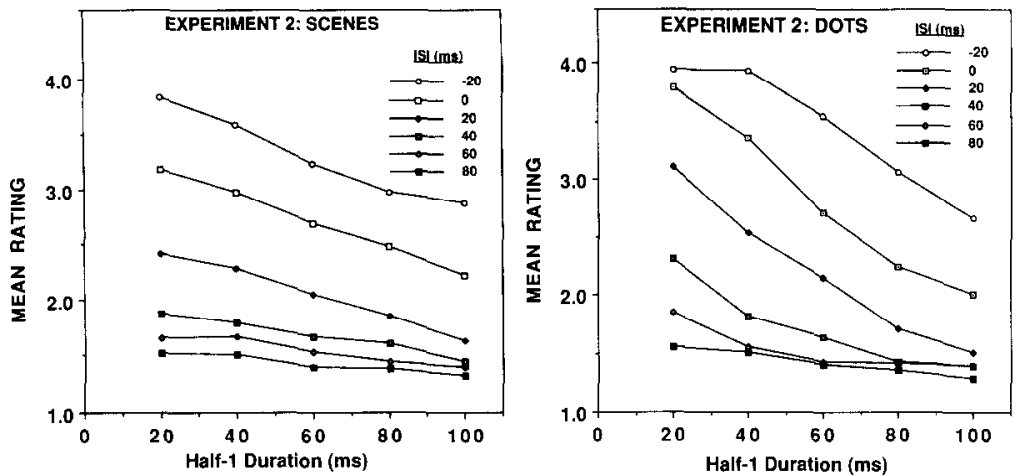

FIG. 3. Experiment 2: Mean rating as functions of half-1 duration. The curve parameter is interstimulus interval. The left panel shows data for scenes; the right panel shows data for dots. Each scene data point is based on 2100 observations, and each dot data point is based on 420 observations.

rating as a function of H1D; a separate curve is drawn for each of the five temporally disjoint ISI levels, plus the synchronized-offset (ISI $=-20$ ) condition. The left panel shows performance averaged over the five scenes, and the right panel shows performance for the dots. Statistical power was again rather high; the standard errors were 0.057 and 0.076 for scenes and dots, respectively.

Temporally disjoint conditions. The Experiment 1 results are replicated quite well; performance for both types of stimuli decreases monotonically with both HID and ISI.

Scenes vs dots. Figure 4, which is organized like Fig. 2, compares the scene and dot performance decreases over HID (left) and ISI (right). In
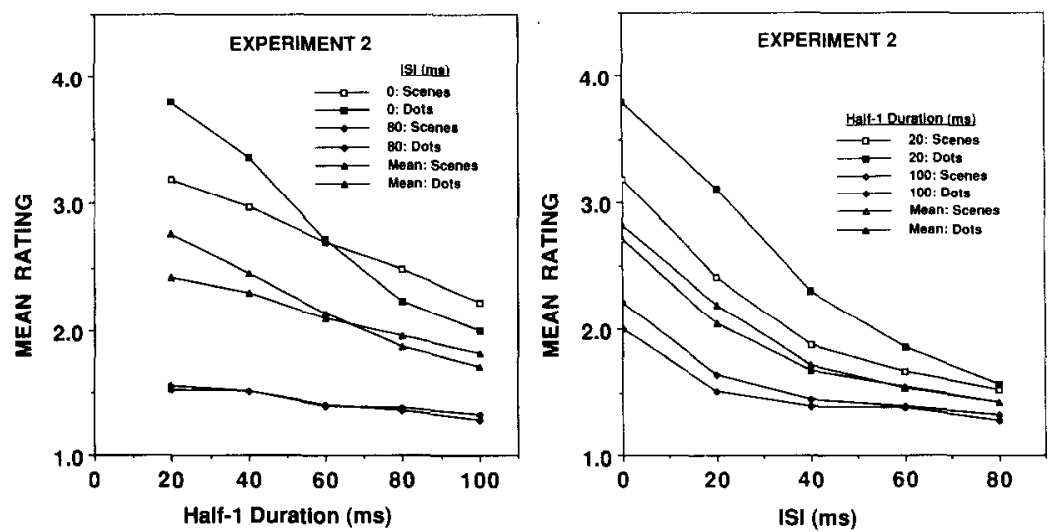

Fig. 4. Experiment 2: The scene/dot $\times$ half- 1 duration interaction for different levels of interstimulus interval (left) and the scene/dot $x$ interstimulus interval interaction for different levels of half-1 duration (right). 
both panels, three pairs of curves are shown: scene and dot performance for the smallest value of the nonabscissa factor (0-ms ISI ${ }^{4}$ or 20-ms H1D), for the largest value of the nonabscissa factor (80-ms ISI or $100-\mathrm{ms}$ half1-duration), and collapsed over the nonabscissa factor (ISI and H1D for the left and right panels respectively). The Experiment 1 pattern is replicated; when the value of either factor is small, dot performance decreases faster than scene performance as a function of the other factor. Again, the scene/dot interaction with either factor disappears when the value of the other factor is large.

As in Experiment 1, we performed a 2 (scene/dot) $\times 5$ (ISI) ANOVA for each of the six H1D levels. Similarly, we performed a 2 (scene/dot) $\times 6$ (H1D) for each of the seven ISI levels. The results of these ANOVAs are shown in Table 2, which is organized like Table 1. As in Experiment 1, the scene/dot $\times$ ISI interaction is significant only for the 20- and 40-ms H1D levels. The scene/dot $\times$ H1D interaction is significant only for the -20 -, $0-, 20-, 40-$, and 60-ms ISI levels.

Temporal-overlap conditions. Figure 5 shows scene and dot performance as functions of H1D for the synchronized-onset conditions (left) and for the synchronized-offset conditions (right). In both conditions, performance falls as a function of H1D. In the synchronized-offset conditions, the relation between scene and dot performance behaves as it does in the temporally disjoint conditions; dot performance falls faster than scene performance. In the synchronized-onset conditions, however, the interaction is reversed; scene performance falls faster than dot performance. Both of these interactions are statistically significant, as indicated in Table 2.

The observers all reported that the synchronized-onset conditions seemed different from the other conditions, and claimed to be making their judgements differently in this condition. Their reports did not suggest an obvious basis for the difference.

Inclusion of the temporal-overlap conditions was motivated by data reported by Di Lollo (1980, Experiment 5). Di Lollo used a forwardmasking paradigm in which a pattern mask was presented for durations ranging from 20 to $660 \mathrm{~ms}$. During the last $20 \mathrm{~ms}$ of mask presentation, a spatially contiguous, to-be-reported target letter appeared. Di Lollo reasoned that, as mask duration increased, encoding activity devoted to it would decrease, thereby rendering the mask less effective in interfering with the target. The results confirmed this reasoning; as mask duration increased from 20 to approximately $160 \mathrm{~ms}$, the probability of correctly reporting the target rose from 20 to over $90 \%$.

\footnotetext{
${ }^{4}$ Technically, the $-20 \mathrm{~ms}$ ISI level is smallest. We used 0 to provide a figure comparable to the corresponding figure in Experiment 1 (Fig. 2).
} 
TABLE 2

Experiment 2: $F$ Ratios for Dot/Scene (D/S) Interactions with Half-1 Durations and ISIs

$\mathrm{D} / \mathrm{S} \times \mathrm{ISI}$ interaction as a function of half-1 duration
$\mathrm{D} / \mathrm{S} \times$ half -1 duration interaction as a function of ISI

\begin{tabular}{crrr}
\hline Half-1 duration $(\mathrm{ms})$ & $F(4,36)$ & ISI $(\mathrm{ms})$ & $F(4,36)$ \\
\cline { 3 - 4 } & & SON & 33.83 \\
20 & 10.56 & SOF: -20 & 10.44 \\
40 & 6.93 & 20 & 20.03 \\
60 & 0.74 & 40 & 22.21 \\
80 & 1.64 & 60 & 11.85 \\
100 & 1.00 & 80 & 3.63 \\
\hline
\end{tabular}

Note. The D/S $\times$ half-1 duration interaction for synchronized-onset (SON) and synchronized-offset (SOF) conditions are also shown. Criterion $F(\alpha=0.05)=2.82$ for $(4,36)$ degrees of freedom.

This fascinating result suggests that temporal separation of two stimuli is not necessary for failure of the two stimuli to integrate. Di Lollo chose to demonstrate this phenomenon using the detrimental effect of masking. Our synchronized-offset data confirm Di Lollo's result using a close analog of the missing-dot task. As indicated in Fig. 5, the synchronizedoffset data are similar to the temporally disjoint data, in that the decline for the dots is greater than the decline for the scenes. There is no evidence in our data for a qualitative difference between a standard, nonnegative ISI condition (say, ISI $=0$ ) and an ISI of -20 , which constitutes the synchronized-offset conditions.
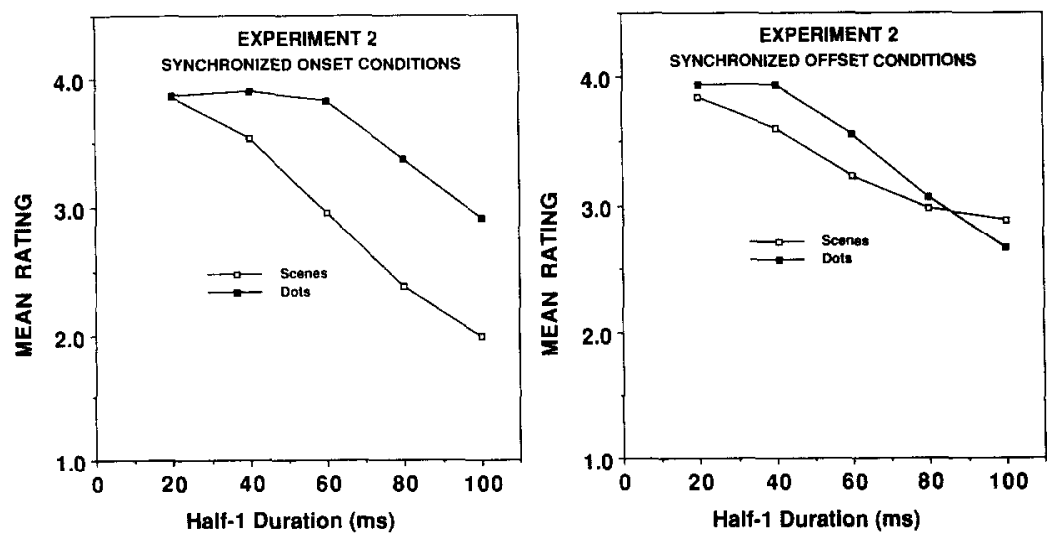

Fig. 5. Experiment 2: Temporal-overlap conditions. Mean rating as a function of half-1 duration for the synchronized-onset conditions (left) and for the synchronized-offset conditions (right). 
In contrast, however, the synchronized-onset conditions behave quite differently from the temporally disjoint conditions. In the dot conditions mean ratings remain at ceiling up to a H1D of $60 \mathrm{~ms}$, and then drop only slightly, while in the scene conditions mean ratings drop continuously as H1D increases from 20 to $100 \mathrm{~ms}$. Thus, the synchronized-onset pattern of results is quite different from the data patterns in all other ISI conditions. As noted above, observers claimed that the synchronized-onset conditions seemed different from the others.

\section{Experiments 1 and 2: Differences in Results}

To assess the relation between the data pattern in Experiment 1 vs Experiment 2, we considered only those conditions that were common to the two experiments: the $3 \times 3$ design consisting of 20, 40, and 80 H1D levels, combined with 0,40 , and 80 ISI levels. We then computed mean rating as a function of ISI, collapsed over H1D, and vice versa. We did this for both scenes and dots, and for both experiments.

The results of this analysis are shown in Fig. 6. In each of the four panels, mean rating is compared under identical circumstances for Experiment 1 and for Experiment 2. The left panels of Fig. 6 show mean ratings as functions of ISI, whereas the right panels show mean ratings as functions of H1D. The top panels show data for scenes, whereas the bottom panels show data for dots.

The data patterns are the same for dots and scenes. First, ratings are generally higher in Experiment 1 than in Experiment 2. This finding could reflect a bias effect; i.e., observers could be using the rating scales somewhat differently in Experiment 1 relative to Experiment 2. Of somewhat greater interest is the Experiment $\times$ ISI interaction indicated in the left panels. This means that the ISI effect on rating is different in the two experiments: it is greater in Experiment 2 than in Experiment 1. We defer additional discussion of these findings until later in this article when we apply a mathematical model to our data.

\section{GENERAL DISCUSSION}

The data obtained in Experiments 1 and 2 allow conclusions about two issues: the relation between temporal-integration and rating performance, and the effects of stimulus complexity on visible persistence.

\section{Completeness Ratings and Temporal-Integration Performance}

A major purpose of the present experiments was to compare the data pattern obtained from the subjective-completeness task with the data pattern obtained from the temporal-integration task. The data patterns are quite similar: a comparison of the present results with those reported by Di Lollo and his colleagues indicates that the effects of both ISI and H1D 

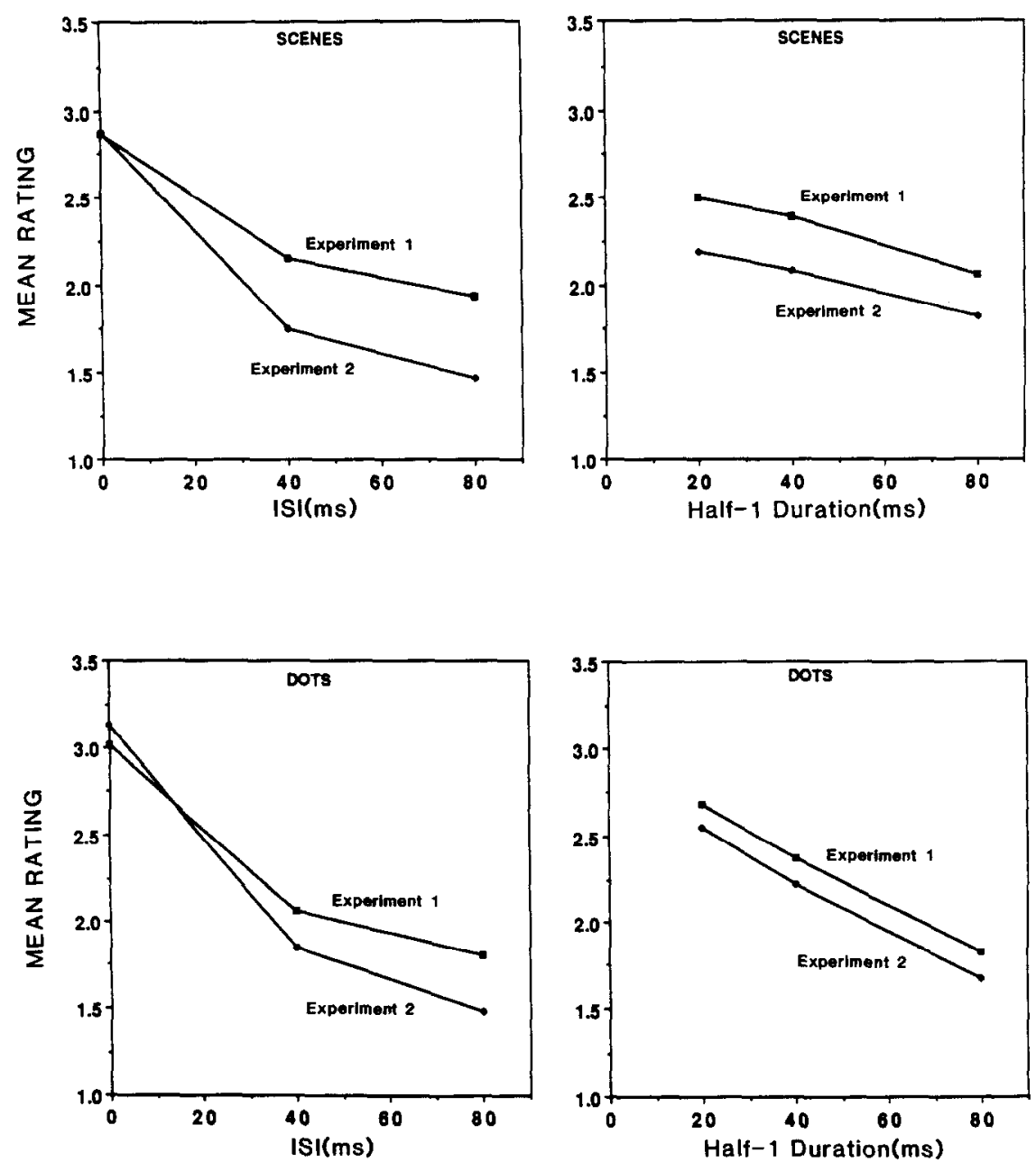

FIG. 6. Experiment 1/Experiment 2 comparisons for conditions that are common to the two experiments. The left panels show rating as functions of interstimulus interval collapsed over half- 1 duration; the right panels show rating as functions of half- 1 duration, collapsed over interstimulus interval. 'The top panels show data for scenes; the bottom panels show data for dots. In all panels, curve parameter is Experiment 1/Experiment 2.

are qualitatively the same on subjective completeness as they are on temporal-integration ability. This similarity supports the general notion that phenomenological appearance and temporal-integration ability are two manifestations of the same perceptual events. The model that we describe below formalizes this proposition.

\section{Scenes vs Dots}

The effects of both H1D and ISI are somewhat different for complex vs 
simple stimuli-at least insofar as complex and simple stimuli are embodied in the scenes and dots that we used in the present experiments. The results shown in Figs. 2 and 4, along with Tables 1 and 2, show that the effect of both factors is more dramatic for simple relative to complex stimuli. This effect has several possible causes.

One such cause is embodied in the model that we shall describe below. More generally, the scene/dot difference supports any theory in which visible persistence is assumed to be determined by degree to which processing of the persisting stimulus is ongoing. By such theories, processing of simple stimuli is completed earlier than processing of more complex stimuli. Thus, processing of simple stimuli is completed both at shorter H1Ds and at shorter ISIs relative to processing of complex stimuli, and persistence declines faster with both of these variables.

\section{AN INFORMATION-EXTRACTION RATE MODEL}

Loftus and Hogden (1988) proposed a model whose major function is to unify information extraction from a visual stimulus on the one hand, and phenomenological awareness of the stimulus on the other hand. Loftus and Hogden (1988), along with Loftus et al. (1988), have successfully applied the model to picture-memory data and to synchrony-judgement data. We will describe the model here and then show how it can be applied to the present data as well as to Di Lollo and Dixon's (in press) partial-report data.

\section{The Model}

Loftus and Hogden were concerned with a situation in which an observer views a briefly presented stimulus with the intention of being able to subsequently remember it. They conceptualize a stimulus as a bundle of information, and the observer's task as extracting as much of the information as possible for transfer to some more permanent memory store. Information is extracted as some rate, $r(t)$, where $t$ refers to time since stimulus onset. Information extraction can occur both during stimulus presence and for a short period following stimulus offset.

A crucial assumption of the model is that information-extraction rate determines visible persistence. This assumption is similar to that made by Di Lollo (1980) who posited visible persistence to be a product of "recruitment activity." In the present model, a stimulus is assumed to remain phenomenologically present until the information-extraction rate falls below some criterion. Generally, the rate does not fall below this criterion until some time following stimulus offset. This is why the stimulus continues to persist phenomenologically following stimulus offset. 


\section{Assumptions}

Loftus and Hogden described their model in terms of four fundamental assumptions, plus a fifth assumption specific to the picture-memory paradigm with which they were concerned. We describe the four fundamental assumptions here. ${ }^{5} \mathrm{~A}$ fifth assumption is designed to apply the model to the present paradigm.

Assumption 1: Available information. A stimulus consists of information that is potentially available to a subsequent information-extraction process. While the stimulus is physically present, all information is available; when the stimulus physically disappears, available information decays over time.

The proportion of total stimulus information available at time $t$ following stimulus onset is designated $a(t)$, the equation for which is

$$
\begin{aligned}
a(t)=1.0 & \text { for } t \leq d \\
e^{-(t-d) / w} & \text { for } t>d,
\end{aligned}
$$

where $d$ is the duration of the stimulus and $w$ is a free parameter, in units of time, estimated by Loftus, Johnson, and Shimamura (1985) to be 100 ms. ${ }^{6}$ Note that the term $(t-d)$ is time since stimulus offset, and corresponds to ISI in the present experimental paradigm. Equation (1) is illustrated in Fig. 7 (top).

Assumption 2: Unidimensionality. Information is unidimensional; that is, both amount of information available in the stimulus and amount of information extracted by the observer can be represented by a single value on some ordinal scale.

Assumption 3: Information-extraction rate. The proportion of total stimulus information extracted by time $t$ is designated $I(t)$. New information is extracted at a rate $r(t)$, where $r(t)$ is the derivative of extracted information with respect to time, i.e., $r(t)=d I / d t$.

The information-extraction rate is assumed to be a product of two factors. The first factor is $a(t)$, the available information (which implies the reasonable property that $r(t)=0$ when $a(t)=0)$. The second factor is a monotonically decreasing function, $h$, of $I(t)$, the proportion of infor-

\footnotetext{
${ }^{5}$ Loftus and Hogden presented two parallel models: one using relatively weak assumptions, and the other using corresponding stronger (quantitative) assumptions. We present only the quantitative version of the model here.

${ }^{6}$ The parameter $w$, termed the icon's worth, is described in detail by Loftus et al. (1985). Briefly, it can be viewed as follows. Loftus et al. found that memory performance for a $d$-ms picture followed by an icon was equal to memory performance for a $(d+100)$-ms picture not followed by an icon. Thus, with respect to memory performance, having an icon is worth having approximately $w=100 \mathrm{~ms}$ of additional physical exposure duration.
} 

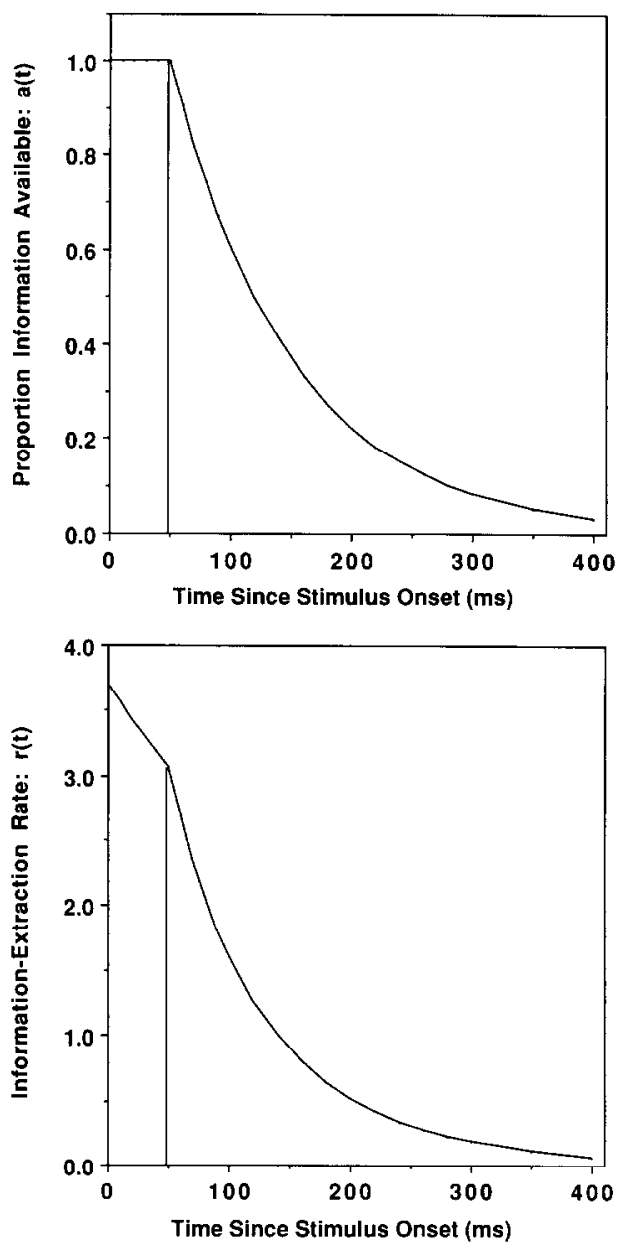

Fig. 7. Major components of the Loftus and Hogden model as functions of time since stimulus onset. The top panel shows available information and the bottom panel shows information-extraction rate.

mation already extracted. That $h(I(t))$ is monotonically decreasing captures the notion that easier (i.e., faster)-to-extract information is extracted earlier than harder (i.e., slower)-to-extract information (just as, for example, the earlier words in a crossword puzzle are filled in faster than the later words). This general characterization of $r(t)$ (in conjunction with unidimensionality) has been incorporated, in one form or another, into a variety of information-acquisition models (e.g., Kowler \& Sperling, 1980; Krumhansl, 1982; Loftus \& Kallman, 1979; Massaro, 1970; Rumelhart, 1969; Sperling, 1967). 
We have chosen the function $h$ to be

$$
h(I(t))=c[1.0-I(t)],
$$

where $c$ is a free parameter, in units of $\sec ^{-1}$, whose value determines how fast $r(t)$ declines with increases in extracted stimulus information. Thus, the equation for $r(t)$ is

$$
r(t)=a(t) c[1.0-I(t)]
$$

Equation (2) is illustrated in Fig. 7 (bottom) with $c=3.70$, a value that was estimated by Loftus and Hogden (1988).

Assumption 4: Phenomenological appearance. An observer remains phenomenologically aware of a stimulus until $r(t)$, the rate of extracting information from the stimulus falls below some criterion, $r_{\text {crit }}$.

An important consequence of this assumption is illustrated in Fig. 8 where $r(t)$ is shown as a function of time since stimulus onset for 50- and 250-ms stimuli. The horizontal line near the abscissa of each graph represents $r_{\text {crit }}$. Duration of visible persistence is represented by the doubleheaded arrows between the time of stimulus offset and the time at which $r(t)$ crosses $r_{\text {crit. }}$. It can be seen that, in accordance with the extant synchrony-judgement data, the model predicts shorter persistence with longer stimuli.

Assumption 4 is essentially that conscious experience of a stimulus results from extracting information from the stimulus. This notion is similar to one in the selective attention literature that conscious experience results from attending to the stimulus (cf. James, 1890/1950; Norman, 1976).

Assumption 5: Rating performance. The completeness rating obtained in the present experiments is a monotonic function of the half- 1 information-extraction rate at the time that the second half of the picture is presented.

Assumption 5 is somewhat arbitrary, but it captures the model's essence, which is that, the lower half- $1 r(t)$ is, the less likely half-1 is to be visible; thus the lower half- $1 r(t)$ is at the time of half-2 onset, the less likely it is that the entire configuration resembles the complete picture. Figure 9 illustrates the model's account of the present data. Each panel shows $r(t)$ for both half- 1 and half- 2 as functions of time since half- 1 onset. The four panels contrast short vs long H1Ds (top vs bottom panels) and short vs long ISIs (left vs right panels). Because half-1 $r(t)$ decreases with both H1D and ISI, the value of half- $1 r(t)$ at the time of half- 2 presentation decreases with both these variables.

\section{Relation to Di Lollo and Dixon's Model}

Earlier, we sketched a visible persistence model described by Di Lollo 

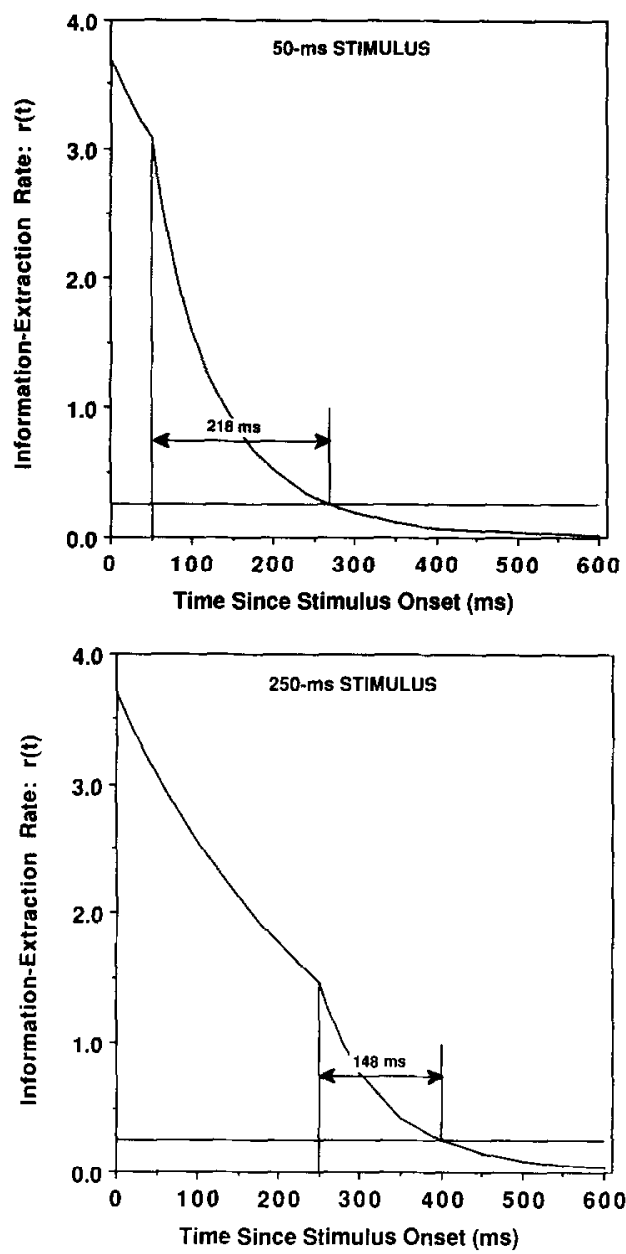

Fig. 8. Prediction of the model about the effect of stimulus duration in a synchronyjudgment task. Each panel shows information-extraction rate as a function of time since stimulus onset. The top panel if for a $50-\mathrm{ms}$ stimulus; the bottom panel is for a $250-\mathrm{ms}$ stimulus. The time for information-extraction rate to fall to a criterion level is greater for the shorter stimulus.

and Dixon (in press). Loftus and Hogden's model is formally quite similar to Di Lollo and Dixon's. Each model involves two major constructs that play corresponding roles. First, Loftus and Hogden posit available information, while Di Lollo and Dixon posit an analog representation. Both constructs are assumed to be (a) time-locked to stimulus offset and (b) not responsible for phenomenological awareness. Second, Loftus and Hogden posit an information-extraction rate, while Di Lollo and Dixon posit 


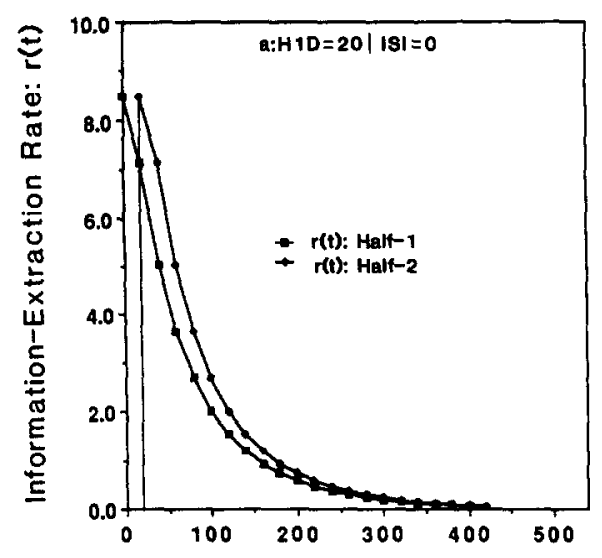

Time Since Half- 1 Onset(ms)

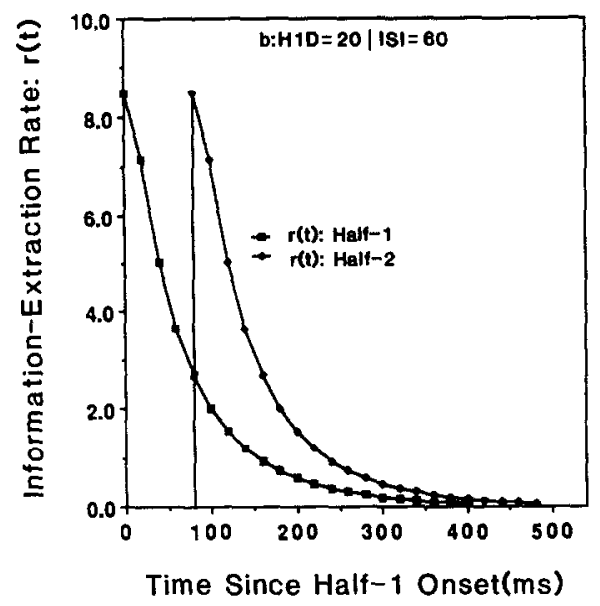

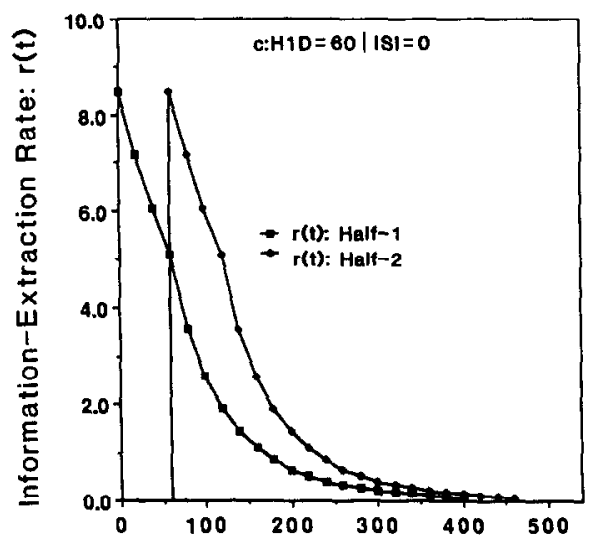

Time Since Half- 1 Onset(ms)

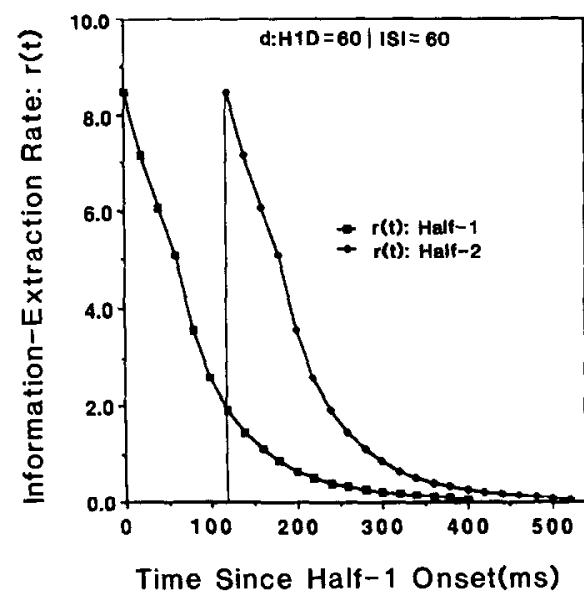

FIG. 9. Illustration of the model's account of the rating task used in Experiments 1 and 2.

visible persistence. Both constructs are assumed to be (a) time-locked to stimulus onset and (b) responsible for phenomenological awareness.

The major difference between the models is in the link between these two constructs and performance in tasks that depend on spatial information. In Di Lollo and Dixon's model, both the analog representation and visible persistence contribute independently to performance. In Loftus and Hogden's model, available information affects performance only indirectly, via its effect on information-extraction rate. 
In the next two sections, we will apply the information-extraction model both to the present data and to Di Lollo and Dixon's (1988) partialreport data.

\section{APPLICATION OF THE INFORMATION-EXTRACTION MODEL TO THE PRESENT DATA}

Equations (1) and (2) allow calculation of the half-1 informationextraction rate at the instant of half-2 presentation for all conditions in both experiments (cf. Fig. 9). We refer to these rates as $r$ (H1D, ISI).

\section{Interpreting the Model's Parameter, $c$}

In the equation for $r(t)$ (Eq. (2)), there is a free parameter, $c$. Because $c$ is important in interpreting the model, we make several remarks about it here.

Rapidity of relevant information extraction. The parameter $c$ determines how fast relevant information is extracted from the stimulus: higher values of $c$ imply quicker extraction of relevant information. Recall that, in our usage, the term "information"-either available or extracted information-refers to proportion of total information in the stimulus. Thus our mathematical expression of information is normalized for stimuli of different informational content. To gain an intuition of $c$ 's meaning, it is useful to calculate how fast some proportion of relevant information is extracted for different $c$ values. Consider, for example, a 20-ms stimulus. With $c=10$, it would require $87 \mathrm{~ms}$ to extract $50 \%$ of relevant stimulus information; with $c=20$, it would require only $36 \mathrm{~ms}$ to extract $50 \%$ of relevant stimulus information.

Stimulus complexity and $c$. The predicted difference between complex and simple stimuli (scenes and dots) is captured in the model by predicted variation in $c$; that is, faster information extraction for dots relative to scenes implies, and is implied by, a higher value of $c$ for dots relative to scenes. In fitting our model, we allowed two free parameters, $c_{\mathrm{s}}$ and $c_{\mathrm{d}}$, in computing the information-extraction rates for scenes and dots, respectively.

\section{Fitting the Model with Rank-Order Correlation}

Because the model assumes performance (rating) to be only monotonic with half-1 $r(t)$, the model cannot precisely predict performance. The monotonicity assumption does, however, predict a perfect over-conditions, rank-order correlation between $r(\mathrm{H} 1 \mathrm{D}, \mathrm{ISI})$ and rating performance, which we refer to as $p(\mathrm{H} 1 \mathrm{D}, \mathrm{ISI})$. Accordingly, to fit the model, we used a gridsearch procedure to find those values of $c_{\mathrm{s}}$ and $c_{\mathrm{d}}$ that maximized the rank-order correlation between $r(\mathrm{H} 1 \mathrm{D}$, ISI) and 
$p$ (H1D, ISI) over experimental conditions. We carried out this procedure separately for Experiments 1 and 2.

\section{Model Fit: Results}

To fit the Experiment 1 data, we used 60 conditions: the six H1D levels crossed with the five temporally disjoint ISI levels for both scenes and dots. The best-fitting $c_{\mathrm{s}}$ and $c_{\mathrm{d}}$ values were 38.5 and 47.5 , respectively, which produced a rank-order correlation, $\rho$ of 0.981 . To fit the Experiment 2 data, we used 50 conditions: the five H1D levels crossed with the five temporally disjoint ISI levels, for both scenes and dots. The bestfitting $c_{\mathrm{s}}$ and $c_{\mathrm{d}}$ levels were 8.5 and 14.5 , respectively, which produced a rank-order correlation, $\rho$, of 0.982 . The results of these fitsover-condition scatterplots relating obtained ratings to predicted $r(t)$-are shown in Fig. $10 .^{7}$ Note that there are separate scatterplots for Experiments 1 and 2 .

It is evident that the fits are reasonably good for both experiments. Could these scatterplots have come from underlying monotonic distributions, as the model predicts? Consider the many reversals in the scatterplots, i.e., pairs of points whose ratings are reversed relative to the model predictions (an example of a reversal is indicated by the two arrows in Fig. 10). If the model is correct, then these reversals must occur by chance. Recall, however, that standard errors are very small-approximately the size of the curve symbols. Accordingly, many of the reversals are several standard deviations apart. In short, the power of the data is such that the observed rank-order correlations, despite being high, are inconsistent with an underlying monotonic fit. This constitutes a deficit of the model about which we will say more shortly.

\section{Model Fit: Discussion}

The model provides a convenient tool within which several facets of the data may be interpreted. We will use the model in particular to interpret the observed differences between the two experiments and the observed differences between scenes and dots.

Differences between Experiments 1 and 2. We know empirically (see Fig. 6) that the same stimulus display leads to a different rating in Experiment 2 relative to Experiment 1. Why does this happen? There are at least two (not mutually exclusive) possibilities. The first is that the same stimulus display leads to a different perceptual event in Experiment 2

\footnotetext{
${ }^{7}$ The $r(t)$ values are shown in ln scales for visual clarity. Note that the ordinal relations under consideration are unaffected by logarithmic (or any other monotonic) transformations.
} 


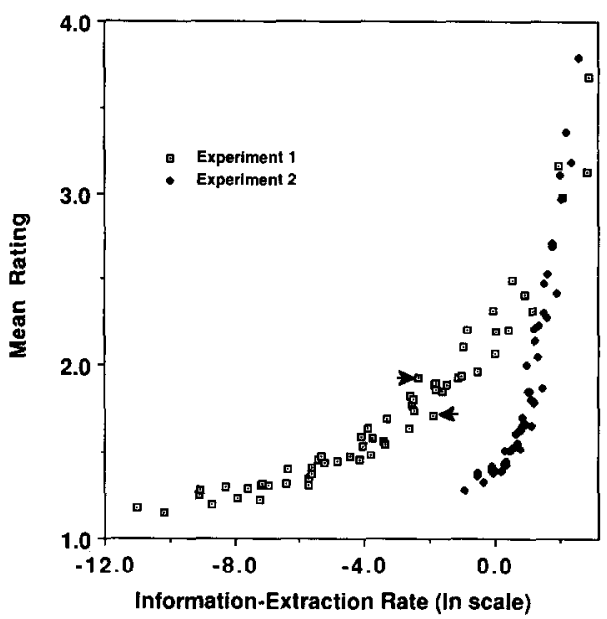

FIG. 10. Fit of the Loftus and Hogden model to Experiment 1 and 2 data. The scatterplots show obtained mean rating as functions of predicted information-extraction rate. Each point on each of the scatterplots represents a single experimental condition. Informationextraction rate is plotted on a natural-log scale for visual clarity. The model's prediction is that all points within each scatterplot form a monotonic function.

than in Experiment 1. The second possibility is that observers are using the rating scale differently in Experiment 2 than in Experiment 1; that is, the same perceptual event may lead to a different completeness rating depending on whether that event occurred as part of Experiment 1 or as part of Experiment 2.

Given the model's validity, both possibilities are correct. Consider first the different best-fitting parameter values in the two experiments. Different parameter values mean that, with the same stimulus display (i.e., with the same H1D and same ISI), $r(t)$ is different in Experiment 2 than in Experiment 1 and, according to the model, different $r(t)$ values imply different perceptual events. But now consider the curves in Fig. 10 that relate observed rating to $r(t)$. The curves are different for the two experiments; e.g., $\ln [r(t)]=0.0$ leads to a rating of about 2.0 for Experiment 1 , but about 1.2 for Experiment 2. Given that the $r(t)$ value specifies the perceptual event, this means that the same perceptual event leads to different ratings in Experiment 2 than in Experiment 1.

That the same perceptual event could lead to different ratings in two experiments seems reasonable; the observers are at liberty to use the rating scale in any way they wish. That the same stimulus display could lead to different perceptual events in the two experiments, while less intuitively plausible, is not out of the question. Suppose that, as asserted by both Di Lollo's (1980) model and Loftus and Hogden's (1988) model, 
the duration of visible persistence depends on the magnitude of perceptual processing. To the degree that magnitude of perceptual processing is influenced by nonstimulus factors, visible persistence would not be strictly determined by stimulus characteristics. In the present Experiment 2, observers were adapted to a different range of H1Ds and ISIs than in Experiment 1. This difference, along with other unknown nonstimulus factors may have influenced perception.

Stimulus complexity. In both experiments, the estimated $c$ value was greater for dots than for scenes. This outcome of the model fit was entirely expected given the data (see Fig. 2 and 4; Tables 1 and 2); it reflects the greater effects of both H1D and ISI on completeness rating for the dots relative to the scenes.

Visual stimuli differ substantially in terms of the amount of relevant information that an observer can extract. Differences in complexity are determined both by the stimulus itself (e.g., a picture containing many different shapes is more complex than a picture containing a single, monochromatic circle) and by what the observer plans to do with the stimulus information (e.g., a picture whose minute details need be remembered is more complex than a picture whose gist only need be remembered).

By the first definition of complexity our naturalistic scenes were more complex than our dot arrays. It is not clear whether the two stimulus types differ by the second definition, because we could not control exactly how the observers were processing the stimuli. In any event, we found scene/dot differences that were quite systematic and robust: dots were more affected by both stimulus duration and by poststimulus duration (ISI) than scenes.

We have provided one explanation for the scene/dot difference that is embodied in our model and is illustrated by the higher $c$ values for dots relative to scenes in both of our experiments. However, as pointed out to us by V. Di Lollo and D. Irwin (personal communication) there are other low-level factors (e.g., contrast, luminance, and spatial-frequency composition) that could also account for the scene/dot difference. We will discuss one such factor that seems particularly plausible, that of contour proximity. We cannot rule out this factor (or any other factor) as being entirely responsible for our observed scene/dot differences. However, as we shall see, if we consider this factor within the context of our model, rather than as an alternative to our model, a tidy and parsimonious picture emerges.

Di Lollo and Hogben $(1985 ; 1987)$ have shown that missing-dot performance is influenced by spatial proximity between the elements of the two successively presented stimulus halves: closer proximity leads to decreased performance at short SOAs. In our experiments, scenes and dots differed with respect to element proximity; the borders of two scene 
halves were spatially contiguous, whereas the half- 1 dots were spatially separated from the half- 2 dots. It is therefore possible that any half-1 visible suppression resulting from half-2 lateral masking is greater at short H1Ds and ISIs for scenes relative to dots. This would result in the shallower slopes for scenes relative to dots that are shown in Figs. 1-4 and Table 1.

To assess this possibility within the context of our model, we plotted mean rating as a function of predicted $r(t)$ separately for scenes and dots. These functions are shown in Figs. 11 (Experiment 1) and 12 (Experiment 2). These figures essentially consist of splittings by scene/dot and ISI of the Fig. 10 scatterplots. Figures 11 and 12 both include six panels. The first five correspond to the five temporally disjoint ISI levels; the data points within each of these five panels correspond to the H1D levels for scenes and dots. The bottom-right panels (labeled "overall") include the same (complete) data as in Fig. 10 (60 conditions for Experiment 1 and 50 conditions for Experiment 2). The model's prediction is that $r(t)$ and rating performance are related by a monotonic function, i.e., that all curves in Figs. 11 and 12 should lie more or less on top of one another. It is evident that this prediction is met reasonably well, although by no means perfectly.

When the scatterplots are arranged this way, systematic departures from monotonicity become apparent. In particular, for all conditions involving short SOAs (shorter than about $60 \mathrm{~ms}$ ), the rating that corresponds to a given $r(t)$ is lower for scenes than for dots. This means that, even after removing the model's account of the scene/dot difference (by generating $r(t)$ predictions based on different $c$ values for scenes and dots), there is still unaccounted scene-dot variation. The nature of this difference-lower ratings for scenes at short SOAs-is exactly what would be predicted by the differential-masking explanation.

We have noted that our two principal variables, H1D and ISI, affect subjective appearance and temporal-integration ability in similar ways. The analysis that we have just reported suggests that a third variablespatial contiguity-also affects subjective appearance and temporalintegration ability in similar ways. This provides additional evidence that subjective appearance and temporal-integration ability are two manifestations of the same perceptual processes.

\section{APPLICATION OF THE INFORMATION-EXTRACTION MODEL TO THE DI LOLLO AND DIXON DATA}

Di Lollo and Dixon (in press) describe a partial-report experiment in which the stimulus display consisted of an array of 15 letters arranged evenly around the circumference of an imaginary circle. The stimulus was presented for one of eight durations, ranging from 10 to $500 \mathrm{~ms}$. Following 

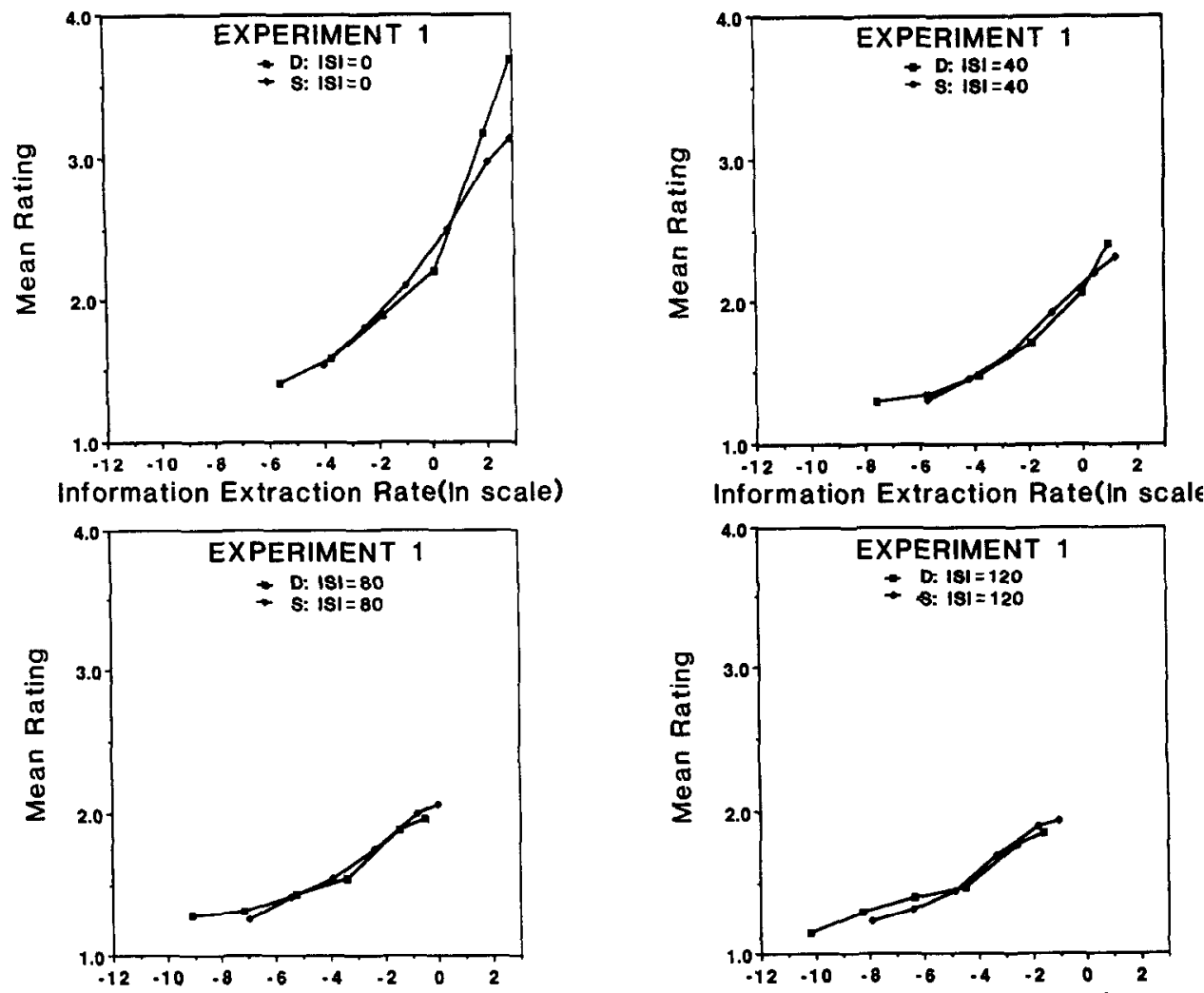

Information Extraction Rate(In scale)
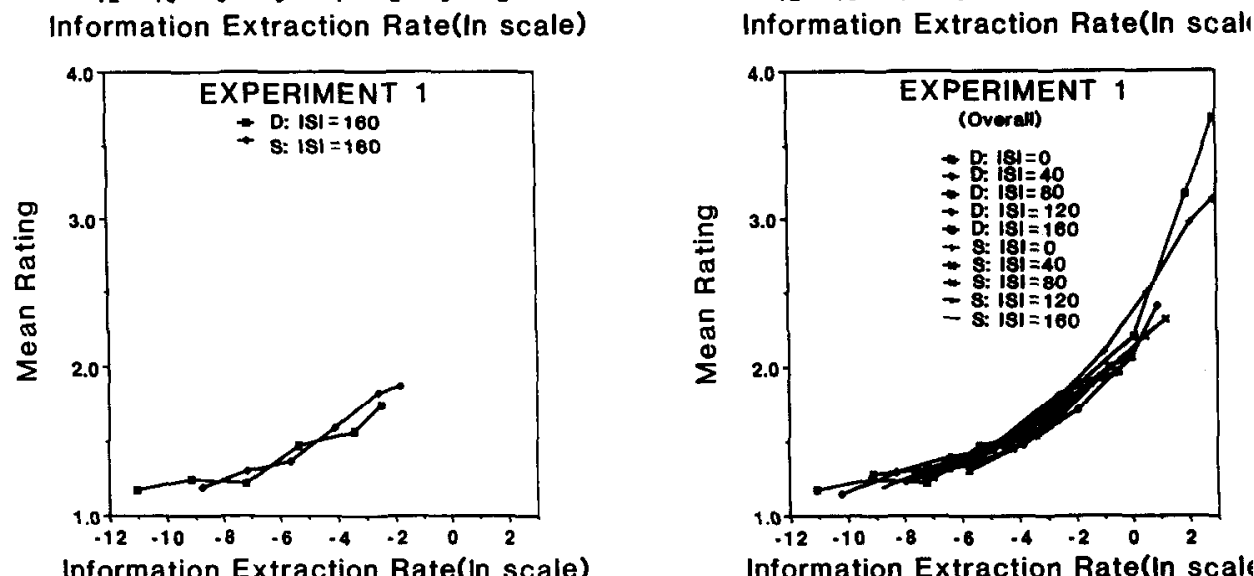

Information Extraction Rate(In scale)

FIG. 11. Fit of the Loftus and Hogden model to data from Experiment 1. Each panel shows observed rating as a function of predicted information-extraction rate; a single point represents a single experimental condition. The bottom right panel shows all 60 conditions. Each of the other panels shows data for one ISI condition only, and includes 12 conditions: six values of $\mathrm{H} 1 \mathrm{D}$ for both scenes and dots. Information-extraction rate is plotted on a natural-log scale for visual clarity. The model's prediction is that all points in a given panel form a monotonic function. 

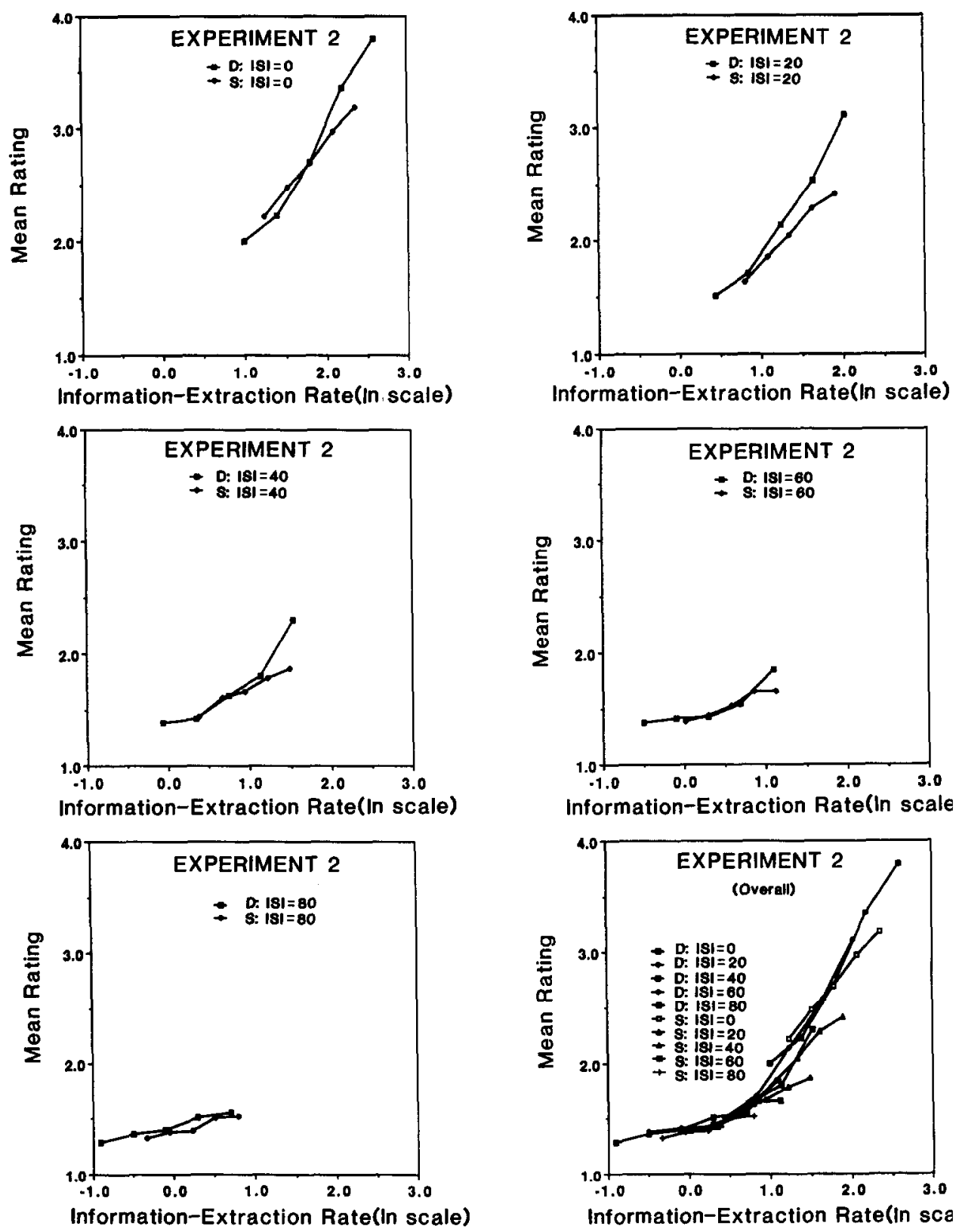

FIG. 12. Fit of the Loftus and Hogden model to data from Experiment 2. Each panel shows observed rating as a function of predicted information-extraction rate; a single point represents a single experimental condition. The bottom right panel shows all 50 conditions. Each of the other panels shows data for one ISI condition only, and includes 10 conditions: five values of $\mathrm{H} I \mathrm{D}$ for hoth scenes and dots. Information-extraction rate is plotted on a natural-log scale for visual clarity. The model's prediction is that all points in a given panel form a monotonic function. 
one of five ISIs, ranging from 0 to $200 \mathrm{~ms}$, a probe appeared in the form of a bar marker indicating one of the letters. The observer's task was to report the indicated letter. Di Lollo and Dixon found that performance varied inversely with both array duration and ISI.

As noted earlier, Di Lollo and Dixon presented a mathematical model to describe their data. This model posits two representations-visible persistence and analog-that contribute independently to performance. The model, which included five free parameters, fit the data impressively, accounting for $99.6 \%$ of the variance across the 40 conditions.

To fit our information-extraction rate model to the Di Lollo and Dixon data, we assumed that information is extracted randomly from the stimulus array up to the point at which the probe is presented. At that point, information extraction continues in the same manner, but is focused on the appropriate array location. Therefore, it is postprobe extracted information that is relevant to subsequent performance. Accordingly, we assume that performance is a monotonic function of the amount of this postprobe extracted information.

Figure 13 illustrates the model's account of Di Lollo and Dixon's data. Each panel shows $r(t)$ as a function of stimulus duration. The four panels contrast short vs long stimulus durations (top vs bottom panels) and short vs long ISIs (left vs right panels). In each panel, the hatched area represents postprobe extracted information (the integral of $r(t)$ from probepresentation position onward). Because $r(t)$ decreases with both H1D and ISI, the magnitude of postprobe extracted information decreases as well.

In fitting our model, we allowed only a single free parameter, $c$. We used a gridsearch procedure to determine the $c$ value that maximized the across-condition rank-order correlation between predicted postprobe extracted information and performance.

\section{Model Fit: Results}

The best-fitting $c$ value was 3.70 , which produced a rank-order correlation, $\rho$, of 0.978 . The fit of the model is shown in Fig. 14, in which mean performance is plotted against predicted postprobe extracted information. ${ }^{8}$ We have plotted separate curves for the separate ISI conditions. There appear to be no systematic deviations from monotonicity.

\section{Model Fit: Discussion}

The information-extraction rate model is capable of accounting for the qualitative pattern of the Di Lollo and Dixon data. We have not predicted exact performance; this would require a theory of the relation between

\footnotetext{
${ }^{8}$ Again, postprobe extracted information is shown on a ln scale for visual clarity.
} 

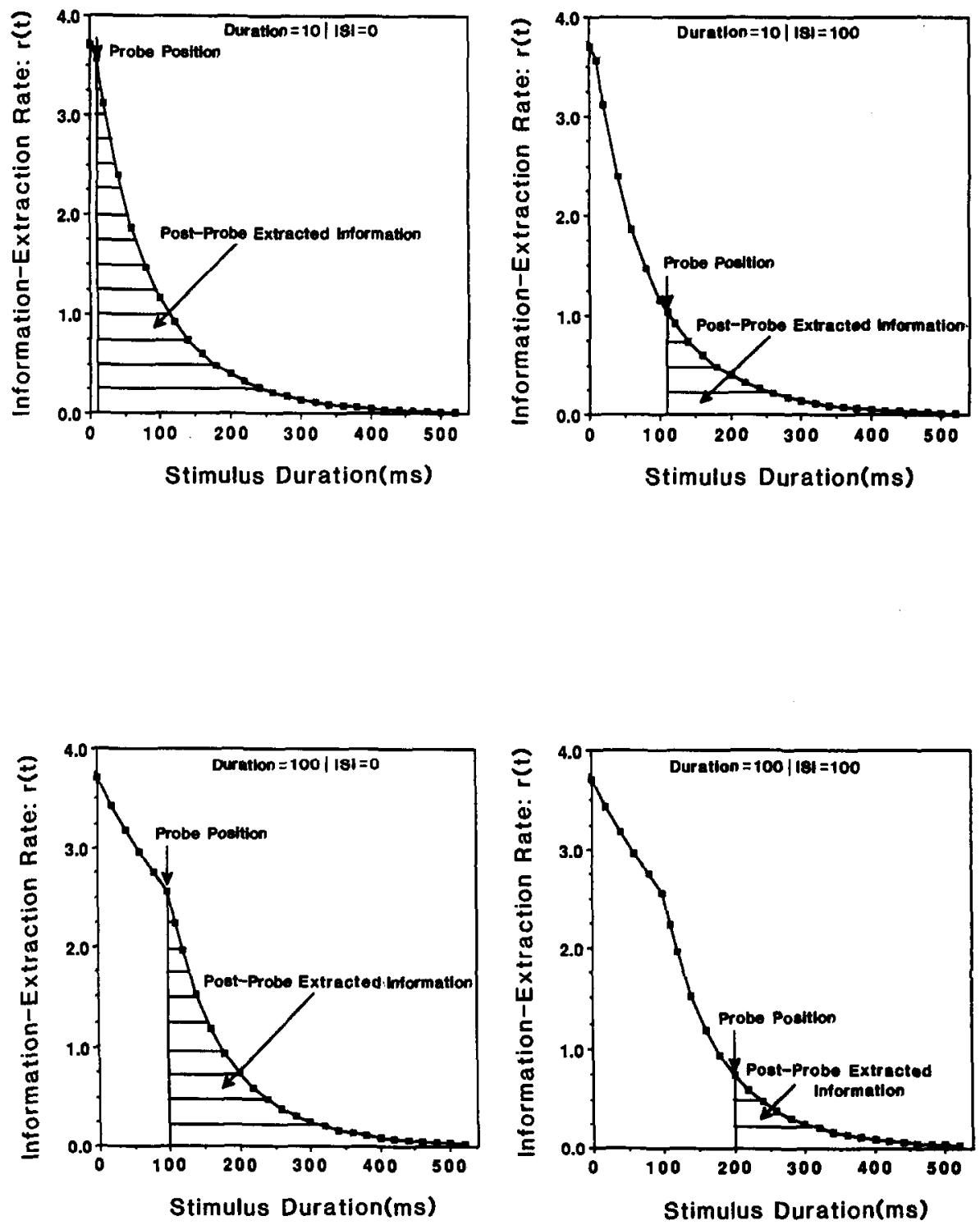

FIG. 13. Illustration of the model's account of the partial- report task used by Di Lollo and Dixon.

postprobe extracted information and actual performance. Development of such a theory is beyond the scope of this article.

Comparison of the models. It is somewhat difficult to compare the accounts of the data provided by the two models. Di Lollo and Dixon 


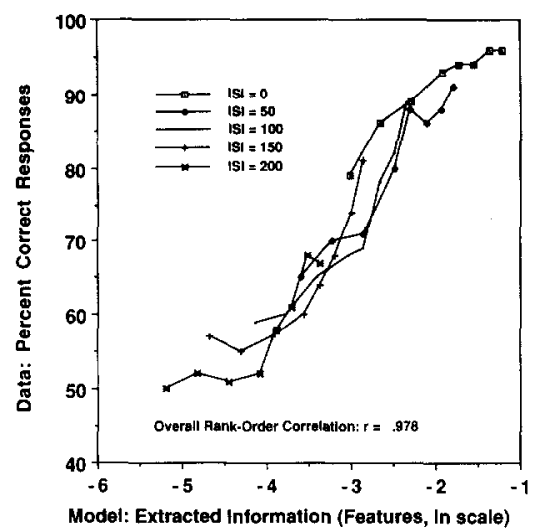

FIG. 14. Fit of the Loftus and Hogden model to Di Lollo and Dixon's data. Each curve shows observed performance as a function of predicted postprobe extracted information for a given ISI level. Extracted information is plotted on a natural-log scale for visual clarity. The model's prediction is that all points form a monotonic function.

predict the quantitative performance pattern very well, whereas we predict the qualitative performance pattern slightly less well. However, Dixon and Di Lollo's model incorporates more free parameters than does ours.

Parameter values. Although we fit our data to the Di Lollo and Dixon data with only one free parameter, $c$, there is another parameter in the model, which is $w$ (see Eq. (1)). Loftus et al. (1985) estimated this parameter to be about $100 \mathrm{~ms}$ and found this value to be remarkably invariant across a variety of conditions. Accordingly Loftus and his colleagues have consistently set $w$ to $100 \mathrm{~ms}$ when applying the Loftus and Hogden model to data (cf. Loftus \& Hogden, 1988; Loftus et al., 1988).

As noted earlier there is a close correspondence between Di Lollo and Dixon's "visual analog representation" and Loftus and Hogden's "available information." Indeed the equations for these two constructs are essentially identical; they are

$$
a(t)=e^{-(i s i) / w} \quad \text { (Loftus \& Hogden) }
$$

and

$$
P(C v a)=a e^{-r(i s i)} \quad \text { (Di Lollo \& Dixon). } .^{9}
$$

Thus there are corresponding parameters in the two models:

${ }^{9}$ Di Lollo and Dixon include the extra parameter, $a$, in their equation to account for contributions to performance from a relatively long-term memory. The decay of the analog representation is entirely captured in the parameter $r$. 


$$
w=1 / r .
$$

Di Lollo and Dixon, using a gridsearch procedure, estimated $r$ to be 0.0105 . Thus $w=1 / 0.0105=95$, which is remarkably close to the $100 \mathrm{~ms}$ estimated by Loftus et al. (1985) and subsequently assumed by Loftus and his colleagues. It is unlikely that Di Lollo and Dixon's fit would have substantially changed had they fixed $r$ at 0.0100 (corresponding to $w=$ 100). Accordingly, in comparing the two models, if one free parameter is to be attributed to the Loftus and Hogden model, only four free parameters should be attributed to the Di Lollo and Dixon model.

\section{CONCLUSIONS}

We conclude with remarks about implications of our data and about illumination of our data that are provided by application of our model.

\section{Remarks about Our Data}

At the outset of this article, we noted that the cognitive activity that briefly follows a visual stimulus has two major characteristics: subjectively, the stimulus itself seems to persist, and objectively, the observer is able to perform tasks that require knowledge of spatial relations within the stimulus. Our first empirical goal was to measure subjective experience in a particular task-the missing-dot task-that was originally designed to measure objective ability.

We found that the result pattern obtained using a subjective measure (completeness ratings) closely resembled previous result patterns obtained using an objective measure (missing-dot performance). This similarity is consistent with the notion that a unitary cognitive phenomenon (information-extraction rate) is responsible for both the subjective and the objective results.

This view differs from that of others (e.g., Di Lollo \& Dixon, in press; Erwin, 1976; Irwin \& Yeomans, 1986) who maintain that spatialintegration ability, while related to visible persistence, is not entirely determined by it. This latter view is expressed most explicitly by Di Lollo and Dixon (in press) who claim performance, at least in a spatially based, partial-report task, to be determined both by visible and by nonvisible stimulus representations.

\section{Remarks about Our Model}

In the second section of this article, we described a model-the Loftus and Hogden model-that has proven capable of accounting for data from a variety of different experimental paradigms (see Loftus \& Hogden, 1988; Loftus et al., 1988, for a complete description of data accounted for by the model). We have applied the model to data from two new tasks: 
our subjective-completeness task and Di Lollo and Dixon's partial-report task. In both cases, the qualitative fit of the model was quite good; the across-condition, rank-order correlations between the model predictions and observed data exceeded 0.97 in all cases.

Of some interest is the nature of the parameter values that emerged from our model fits. In past work, Loftus and his colleagues have obtained best-filling $c$ values of 3.70 from synchrony-judgement data (Loftus \& Hogden, 1988) and 3.40 from picture-recognition data (Loftus et al., 1988). As noted earlier, we found a best-fitting $c$ value of 3.70 in applying our model to Di Lollo and Dixon's partial-report data. In contrast, application of the model to the present data-particularly to the Expcriment 1 data-produced much higher $c$ values. Why is this?

As we have described, high $c$ values are generally associated with rapid extraction of relevant information. Rapid information extraction can occur either because the stimuli are intrinsically simple, or because the observer is treating the stimuli in a simple manner. ${ }^{10}$

In the experiments that have produced low $c$ values, the observers were relatively unfamiliar with the stimuli. In contrast, in the present experiments, observers had seen each picture 216 times during the practice session before Experiment 1 even began; thus, during the experiments themselves, observers were probably learning very little about the pictures that they did not already know. They were quite familiar with the stimuli and might have begun treating them more as random patterns than as coherent stimuli. Thus the relevant information in the stimuli extracted by the observers may have been minimal.

Given this analysis, there is still a mystery: why were the best-fitting parameter values lower in Experiment 2 than in Experiment 1? While we have no compelling answer to this question, we can offer the following speculative analysis. First, given that observers had seen each stimulus 216 times by the beginning of Experiment 1, it is unlikely that there would have been any additional increase in stimulus familiarity as a result of actually participating in the Experiment 1 and Experiment 2 experimental trials. However, there was an important difference between the two experiments: both H1D and ISI were long in Experiment 1 relative to Ex-

\footnotetext{
${ }^{10}$ Loftus et al. (1988) also fit data from several picture-recognition experiments reported by Intraub and her colleagues. They estimated $c$ to be 13.10 for data reported by Intraub (1980) and 10.30 and 5.70 for simple and complex pictures reported by Intraub and Nicklos (1981). In the Intraub (1980) experiment, a picture-recognition paradigm was used in which simple targets and distractors were made to be very different from one another; thus, the task-relevant information that needed to be extracted was minimal. In the Intraub and Nicklos (1981) experiment, the simple pictures were much like those of Intraub (1980) whereas the complex pictures were much like those used by Loftus and Hogden.
} 
periment 2, which led to relatively incomplete perceptions of the pictures in Experiment 1 relative to Experiment 2. Accordingly, observers in Experiment 1 may have been more inclined to treat the pictures as meaningless patterns relative to Experiment 2; on most Experiment 2 trials, the display in fact looked like a fairly normal picture. In short, the different general environment in the two experiments may have produced different ways of processing the pictures on a trial-to-trial basis.

In applying the model to data, we have resisted formulating a complete response model designed to explicitly link the model to observed performance. This approach has advantages and disadvantages. The major disadvantages are (1) we are left with an incomplete account of experimental data and (2) we are restricted to rank-order correlation methods in fitting and evaluating the model. The major advantage is that we do not tie ourselves to restrictive, and probably untenable assumptions about response processes: accordingly we can examine what is of most interest to us-perceptual processes-in relative isolation.

\section{REFERENCES}

Bowen, R. W., Pola, J., \& Matin, L. (1974). Visual persistence: Effects of flash luminance, duration and energy. Vision Research, 14, 295-303.

Coltheart, M. (1980). Iconic memory and visible persistence. Perception and Psychophysics, 27, 183-228.

Di Lollo, V. (1980). Temporal integration in visual memory. Journal of Experimental Psychology: General, 109, 75-97.

Di Lollo, V., \& Dixon, P. (in press). Two forms of persistence in visual information processing. Journal of Experimental Psychology: Human Perception and Performance.

Di Lollo, V., \& Hogben, J. H. (1985). Suppression of visible persistence. Journal of Experimental Psychology: Human Perception and Performance, 11, 304-316.

Di Lollo, V., \& Hogben, J. H. (1987). Suppression of visible persistence as a function of spatial separation between inducing stimuli. Perception and Psychophysics, 41, 345354.

Di Lollo, V., \& Woods, E. (1981). Duration of visible persistence in relation to range of spatial frequencies. Journal of Experimental Psychology: Human Perception and Performance, 7, 754-769.

Efron, R. (1970a). The relationship between the duration of a stimulus and the duration of a perception. Neuropsychologia, 8, 37-55.

Efron, R. (1970b). Effect of stimulus duration on perceptual onset and offset latencies. Perception and Psychophysics, 8, 231-234.

Eriksen, C. W., \& Collins, J. F. (1967). Some temporal characteristics of visual pattern perception. Journal of Experimental Psychology, 74, 476-484.

Erwin, D. E. (1976). Further evidence for two components in visual persistence. Journal of Experimental Psychology: Human Perception and Performance, 2, 191-209.

Hogben, J. H., \& Di Lollo, V. (1974). Perceptual integration and perceptual segregation of brief visual stimuli. Vision Research, 14, 1059-1069.

Intraub, H. (1980). Presentation rate and the representation of briefly glimpsed pictures in memory. Journal of Experimental Psychology: Human Learning and Memory, 6, 1-12. 
Intraub, H. (1985). Visual dissociation: An illusory conjunction of pictures and forms. Journal of Experimental Psychology: Human Perception and Performance, 11, 431442.

Intraub, H., \& Nicklos, S. (1981). The capacity of short-term memory for successively presented pictures. Paper presented at the APA annual convention, Los Angeles.

Irwin, D. E., \& Brown, J. S. (in press). Tests of a model of informational persistence. Canadian Journal of Psychology.

Irwin, D. E., \& Yeomans, J. M. (1986). Sensory registration and informational persistence. Journal of Experimental Psychology: Human Perception and Performance, 12, $343-$ 360.

James, W. (1950). The principles of psychology. New York: Dover (Original work published 1890 ).

Kowler, E., \& Sperling, G. (1980). Transient stimulation does not aid visual search: Implications for the role of saccades. Perception and Psychophysics, 27, 1-10.

Krumhansi, C. (1982). Abrupt changes in visual stimulation enhance processing of form and location information. Perception and Psychophysics, 32, 511-523.

Loftus, G. R. (1985). On worthwhile icons: Replies to Di Lollo and Haber. Journal of Experimental Psychology: Human Perception and Performance, 11, 384-388.

Loftus, G. R., Gillispie, S., Tigre, R. A., \& Nelson, W. W. (1984). A computerized slideprojector laboratory. Behavior Research Methods, Instrumentation, and Computers, $16,447-453$.

Loftus, G. R., Hanna, A., \& Lester, L. (1988). How one picture captures attention from another picture. Cognitive Psychology, 20, 237-282.

Loftus, G. R., \& Hogden, J. (1988). Picture perception: Information extraction and phenomenological appearance. In G. H. Bower (Ed.), The psychology of learning and motivation (Vol. 22). San Diego, Academic Press.

Loftus, G. R., Johnson, C. A., \& Shimamura, A. P. (1985). How much is an icon worth? Journal of Experimental Psychology: Human Perception and Performance, 11, 1-13.

Loftus, G. R., \& Kallman, H. (1979). Encoding and use of detail information in picture recognition. Journal of Experimental Psychology: Human Learning and Memory, 5, 197-211.

Massaro, D. W. (1970). Perceptual processes and forgetting in memory tasks. Psychological Review, 77, 557-567.

Norman, D. A. (1976). Memory and attention. New York: Wiley.

Potter, M. C. (1976). Short-term conceptual memory for pictures. Journal of Experimental Psychology: Human Learning and Memory, 2, 509-522.

Rumelhart, D. E. (1969). $\Lambda$ multicomponent theory of the perception of bricfly cxposed visual displays. Journal of Mathematical Psychology, 7, 191-218.

Sakitt, B. (1976). Iconic memory. Psychological Review, 83, 257-276.

Sperling, G. (1960). The information available in brief visual presentations. Psychological Monographs, 74, 1-29.

Sperling, G. (1967). Successive approximations to a model for short-term memory. Acta Psychologica, 27, 285-292.

Yeomans, J. M., \& Irwin, D. E. (1986). Stimulus duration and partial report performance. Perception and Psychophysics, 37, 163-169.

(Accepted December 12, 1988) 